\title{
The Function of Achieving the SDN Based on the Ethernet and the Open Flow Network
}

\author{
Min Chen, Jing- guo Zhao*, Ze-jun Li and Ang Li \\ School of Computer Science and Technology, Hunan Institute of Technology, Hengyang, China
}

\begin{abstract}
Recently, the SDN is a popular research field. The centralized controller can control all network devices. In addition, the priority if the packet transmitting route of the network or the supervision of the network safety can be conducted from the comprehensive angle. The ECOE proposed in the paper is a kind of the network model of the SDN. It can support many important functions in the SDN by deploying the Ethernet switch in the core network and the OpenFlow switch at the network edge. This paper will introduce how ECOE network model can control the flow-based routing for different kinds of packets. In addition, it can flexibly limit the broadcast/multicast domain and dynamically set the flow load balance to the whole network. Finally, it can also explains how the structure of the ECOE supports the functions of the Service Chain in the Network Function Virtualization (NFV).
\end{abstract}

Keywords: Software-defined networking, SDN, hybrid software-defined networking, network function virtualization, nfv, service chain.

\section{INTRODUCTION}

The SDN is the hot research topic at present and can control all network devices through the centralized Controller instead of adopting the distributed algorithms to determine the behavior of the network packet flow. In this way, the routing of each packet can be set so that the network efficiency can be improved, the network service nodes can be determined for each packet and the function of the network service chain can be provided.

The SDN can develop the network control software in the Controller and set the Openflow switch through the Openflow protocol for achieving the request of the SDN. The Openflow is a kind of the communication protocol between the Controller and the network equipment and the protocol can make the network equipment modify and transfer the packet contents according to the information from the second layer to the fourth layer. Compared with the general switch and the routers, it has more flexible and can largely improve the control-ability of the network. The Controller can give many rules for the network devices through the Openflow protocol. The network devices can do the corresponding actions to the packets in terms of these rules so that the centralized Controller can control the target of the whole network.

$\mathrm{T}$ Although the there is more flexibility by the equipment of the Openflow to the solution of the packets, the present network devices are mainly based on the Ethernet equipment. If all devices are changed into the Openflow equipment for using the SDN, the cost will become much higher. The ability of transmitting the packets in the present

*Address correspondence to these authors at Hunan Institute of Technology, Hengyang, China; Tel: +008613875609142; Fax: +0086734-3452076; E-mails: 445283431@qq.com
Ethernet devices has excellent efficiency. If you can make use of the high flexibility of the Openflow equipment and the ability of rapidly transmitting the packets in the Ethernet devices. The advantages brought by the SDN under the present situation can be enjoyed.

The network model of the Ethernet-in-Core OpenFlowat-Edge (ECOE) is proposed in the paper and a part of the core network adopts the traditional Ethernet switch and the Openflow equipment is used in the edge of the network so that the majority of the important functions in the SDN can be provided.

\section{RELATED RESEARCHES}

\subsection{Ethernet SDN}

Before the Openflow is discussed, the Engineering research center of the cloud has taken up studying the SDN in the Ethernet. The Peregrine adopts the environment of the Ethernet to develop the Network Virtualization, the Dynamic Traffic Engineering and the Fast Failover. It proves that the function of the SDN can also be conducted under the environment of the Ethernet, while the Openflow is just a kind of method of achieving the SDN but not the only one. There is no denying that the Openflow can largely improve the controlling ability of the network equipment by the network control center. Therefore, how to connect the flexibility of the Openflow and the present Ethernet is an important research issue.

\subsection{SDN Controller}

When the Openflow appears, there are many controllers of the Openflow, such as NOX [1-5] and Floodlight [6]. 


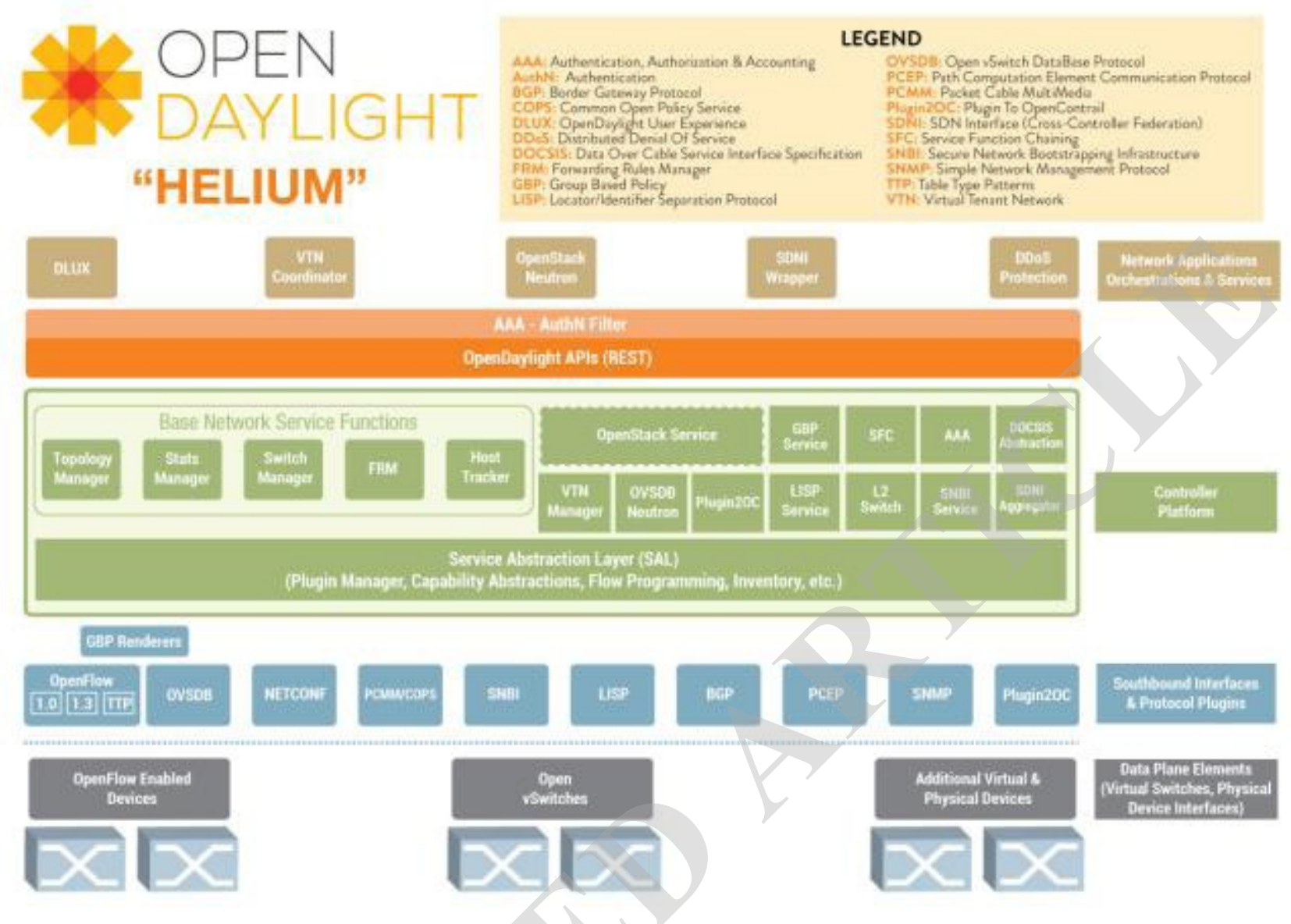

Fig. (1). The framework of the OpenDaylight.

These controllers is mainly based on controlling the Openflow equipment. The OpenDaylight controller is launched by the Cisco on April, 2014. The controller not only controls the Openflow switch through the Openflow protocol but also the bottom of the network equipment through other protocols. The idea is different from it proposed by the Engineering research center of the cloud. Therefore, the SNMP4SDN [7$8]$ in the OpenDaylight is proposed by the Engineering research center of the cloud on April, 2013. The Ethernet equipment can also be added into the SDN control by controlling the Ethernet devices with the use of the SNMP and the CLI orders.

The Fig. (1) is the framework of the OpenDaylight, and the network service procedure of the controller can control the bottom of network devices through the SAL. The SAL layer can set the network devices in terms of the Openflow device or the Ethernet device with the use of different protocols. In this way, the Openflow device or the Ethernet device can be managed by the same controller and the function of the SDN can be offered.

\section{THE NETWORK FRAMEWORK OF THE ECOE}

\subsection{The Framework of the ECOE}

The framework of the ECOE is as shown in the Fig. (2), and the equipment with the ability of the OpenFlow is used on the edge of the network, such as the OpenFlow route, the wireless base station of the OpenFlow or the Open vSwitchn installed in the $\mathrm{x} 86$ host (the virtual switch of the software supporting the Openflow protocol). A part of the core network adopts to the general Ethernet switch. The controller can control the Ethernet switch of the core network and the Openflow device on the edge of the network can offer the function of the SDN.

\subsection{The Technological Features and the Theoretical Ba- sis of the ECOE}

The edge of the network adopts to the equipment with the Openflow ability under the ECOE environment. All the packets can pass the two Openflow equipment during the process of passing the ECOE environment. One is called as the Openflow device of the source port. The other is called as the Openflow device of the terminal port. There are three stages. The first stage is that the packets will pass the Openflow device of the source port during the process of transmitting the packets to the ECOE environment. The second stage is that the packets will flow from the Openflow device of the source port to the traditional Ethernet device in the core network. All packets will be transmitted to the other port of the Openflow device in many Ethernet devices. The third stage is that the the packets will flow from the Openflow device of the terminal port to the traditional Ethernet device. The oper- 


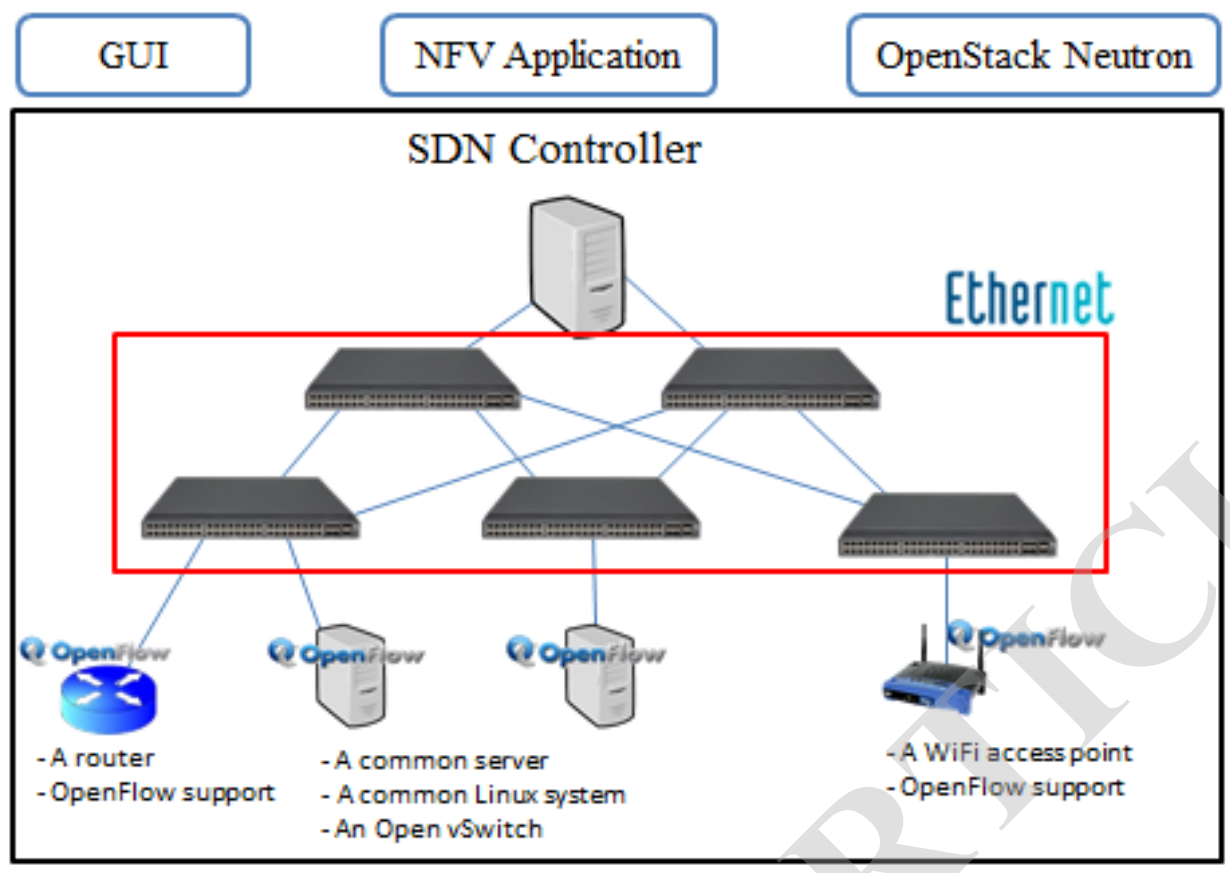

Fig. (2). The framework of the ECOE.

ations of the three stages will be introduced in detail in the followings.

The packets will pass the Openflow device of the source port during the process of transmitting the packets to the ECOE environment in the first stage. Compared with the trasitional Ethernet device, the Openflow device can see many marks of packts and the contents of the packets can be modified. Therefore, the flexible matching rules in the Openflow device of the source port can be set and a special ID can be endowed in the matching rule unde the process of designing ECOE. In addition, the ID should be set in the address of the target MAC of the packets. Later, the packets should be transmitted in the Etjernet of the core network. For example, when the target number of the TCP is equal to the 5601 packet, the address of the target MAC is set as flow 1 and the packet should be transmitted into the core network.

When the core network receives the packets sent by the source port of the Openflow device in the second stage, the modified address of the target MAC has ben changed into a certain flow ID and the ID should be accorded with the MAC form with the unicast for the core network is the traditional Ethernet switch. The traditional Ethernet switch transmits the packets in terms of the address of the target MAC and the controller can set the Static Forwarding Table of the Ethernet switch for the MAC address with the use of the flow ID. Therefore, the Ethernt switch can transmit the pakcets in terms of the corresponding ports of the flow ID in the Static Forwarding Table, the transmitting route of the poackets can be conrtolled if the controller canset the Static Forwarding Table of the Ethernet switch along the core network. At last, it can be sent to the terminal port of the Openflow equipment. Although the Ethernet switch transmits the packets according to the ratget $\mathrm{MAC}$, it seems that the Etehrnet switch can do the packets transmission in terms of a certain Openflow matching rule and the Etehrnet can be directly appplied in the SDN for the target MAC has replaces a certain ID and the flow ID represents a certain Openflow matching rules.

When the terminal port of the Openflow device receives the packets of a certain flow ID from the target MAC address transmitted by the Ethernet switch in the third stage, the rules of the terminal port of the Openflow device will revert the packets into the contents of the original target MAC amd the packets should be transmitted judged by which flow ID should be chosed by the target MAC address of the receiving packets .

The form revesion of the whole packet is as shown in the Fig. (3). When the VM2 transmits the packet to the VM2, there is a TCP online and the number of the target ports in the TCP is 5601. The address of the target MAC is the MAC addressof the VM2 for the packets will be transmitted to the VM2. When the packets get to the source port of the Openflow device, there is a matching rule in the Openflow device. If the port number of the target is the packet of 5601, the target MAC address of the packet can be modified into the flow 1 and the packet should be transmitted to the core network. When the Ethernet switch of the core network transmit the packets to the terminal port of the Openflow device according to the setting Static Forwarding Table, there is a matching rule in the terminal port of the Openflow device. If the target MAC address is the packet of the flow1, the target MAC address should be changed into the MAC address of the VM2, and the packets should be transmitted to the ports of the VM2. Later, the VM2 can receive the packet, but the 


\title{
- Edge OpenFlow switch
}

\author{
- Source OpenFlow switch \\ - Traffic classifier \\ - Encode flow ID to destination MAC address
}

\section{- Destination OpenFlow switch}

- Correct the destination MAC address according to flow ID

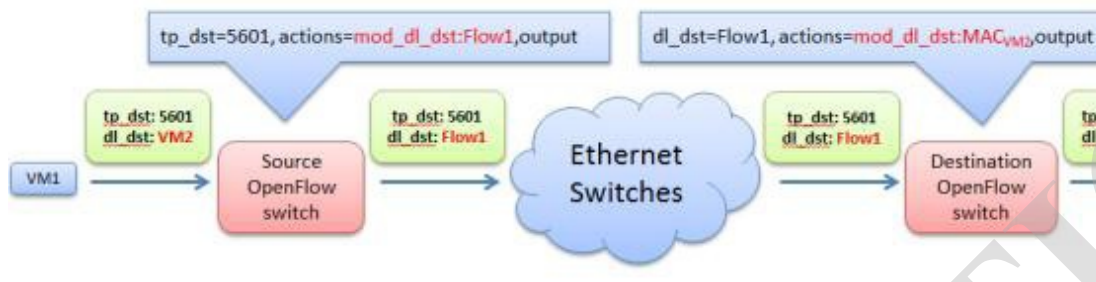

Fig. (3). The operation principle of the ECOE.

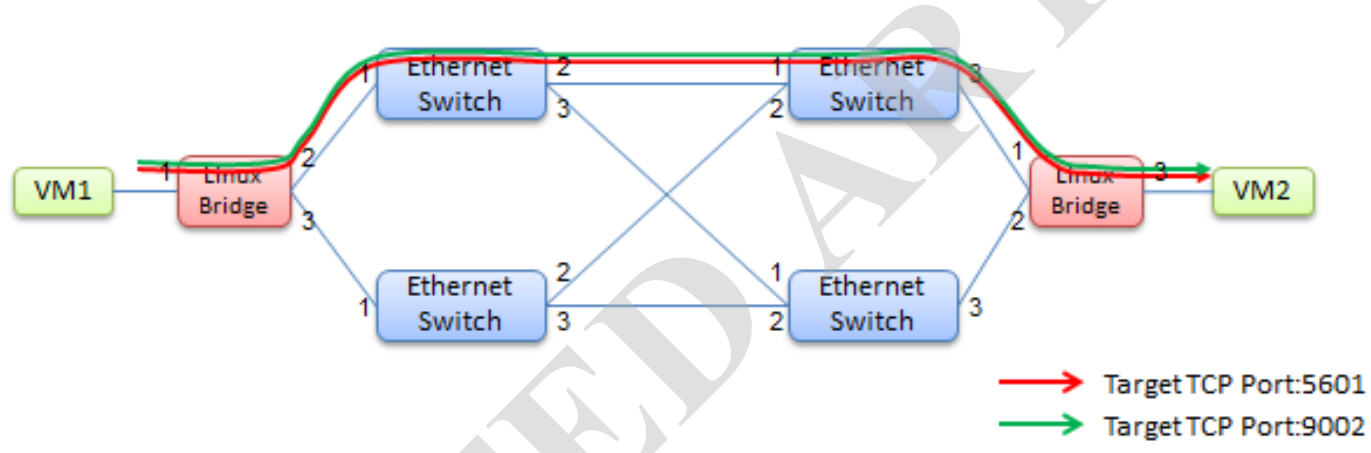

Fig. (4). The Ethernet can do the unicast in terms of the Target MAC address.

VM2 does not know that the passing ECOE environment by the packets is the reversion of the packet contents and the procedure of the transmission.

Although the traditional Ethernet swithc transmits the packets just in terms of the target MAC address, the advantages of the flexible matching rules retrieved by the Openflow device can be obtained with the use of the above methods. The target MAC address of the packets which have been matched can replace a certain ID and it can be transmitted to the Ethernet environment so that the traditional Ethernet switch can also transmit the packets in terms of the matching rules. It seems that the Openflow possesses the transmitting ability in terms of the differnet mathicng rules.

The following parts will introduce how the ECOE can offer the functions provided by the Openflow SDN.

\subsection{The Function of the ECOE SDN}

\subsubsection{Unicast Flow Routing}

The Fig. (4) is the diagram of transmitting the packets through the traditional Ethernet switch. The VM1 and the
VM2 have two TCP onlines, and the number of the target points in the TCP is 5601 and 9002 respectively. Although the number of the TCP target ports whose packets are sent to the VMW by the VM1 is different, the address of the target MAC is the MAC of the VM2 for the traditional Ethernet switch transmits the packets in terms of the target MAC address. In this way, the Ethernet switch can transmit the packets according to the MAC address so that the two types of the packets just can pass the same route. What's worse, the Ethernet switch can not identify the two packets and the two kinds of the packets should be distributed.

The Fig. (5) adopts the Openflow devices to do the transmission of the packet distribution for the Openflow switch can see many segments of the packets. Therefore, the Openflow rules can do the distrubution in terms of the two different kinds of the packets 5601 and 9002 . There are two rules on the left of the OVS (Open vSwitch is a software of the OpenFlowdevice)in the Fig. (5). The first rule is that the target ports of the TCP is the packets of 5601, and it should be sent to the 2 port of the switch. The second rule is that the target ports of the TCP is the packets of 9002, and it should be sent to the 3 port of the switch. While other Openflow 


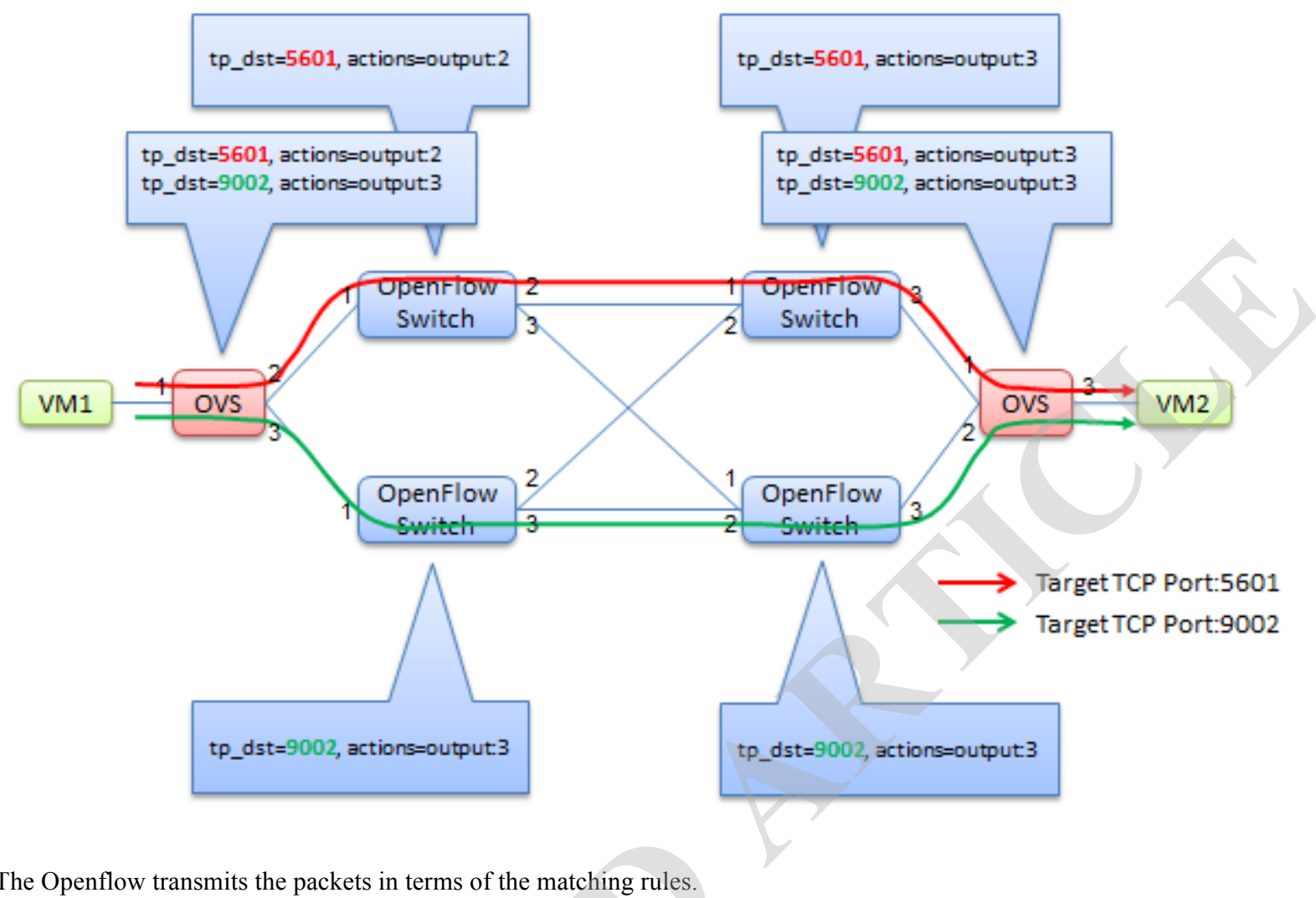

Fig. (5). The Openflow transmits the packets in terms of the matching rules

devices in the core network transmit the packets according to the target port number of the TCP. Finally, the packets should be transmitted to the VM2 for each Openflow device can identify the two kinds of packets, 5601 and9002. Therefore, different routing transmission can be done for achieving the function of the packet transmitting distribution.

The Fig. (6) adopts the ECOE environment to reach the transmitting ability of the packet distribution and the edge Openflow can be adopted to do the matching rules and endowed with a single flow ID in the ECOE environment. There are two rules on the left of the OVS. The first rule is that the target MAC address can be modified into flow1 when the target TCP port is 5601 and it should be sent to the swicth port 2.The second rule is that the target MAC address can be modified into flow2 when the target TCP port is 9002 and it should be sent to the swicth port 2. The Ethernet switch in the core network can do the transmission of different routes in terms of the flow 1 or the flow 2 so that the function of the packet transmitting distribution can be obtained. The OVS on the right, that is the terminal port of the Openflow device, has to revert the flow ID into the original target MAC address. Therefore, there are also two rules on the right of the OVS. The first rule is that the target MAC address should be modified into the VM2 and transmitted to the switch 3 when thetarget MAC address is flow1. The second rule is that the target MAC address should be modified into the VM2 and transmitted to the switch 3 when thetarget MAC address is flow2.

\subsubsection{The Load Balance of the Dynamic Flow}

The load balance of the dynamic flow is an important function in the SDN and the function can also be obtained in the ECOE framework. There are two different onlines in the Fig. (7), that is, the TCP target port 5601 and 9002. The two packets pass the same Ethernet switch originally. When the two onlines do a large number of packet transmissions, the limited bandwidth can be competed and the bottleneck of the network efficiency can happen to the Ethernet switch.

The controller needs to set the load balance of the dynamic flow. The target MAC address can be changed into the flow 1 and the flow 2 for the target TCP port is 5601 and 9002. There fore, the controller just needs to set the contents of the Static Forwarding Table of the flow1 and the flow2 in the Ethernet switch, as shown in the Fig. (8). The two TCP onlines can be distributed si that the network transmitting efficiency can be improved.

\subsubsection{The Range Control of the Broadcast/Multicast}

Many groups of the broadcast/multicast can also be set in the ECOE network. The VM1, the VM2 and the VM4 belong to the same multicast while the VM3 does not belong to the multicast in the Fig. (9). The multicast rules in the edge Openflow of the ECOE should be set. The first rule is that the corresponding VLAN(Virtual LAN) ID should be added in the packet when the multicast network address is 226.139.1.2 so that the pakcet should have the VLAN ID, 


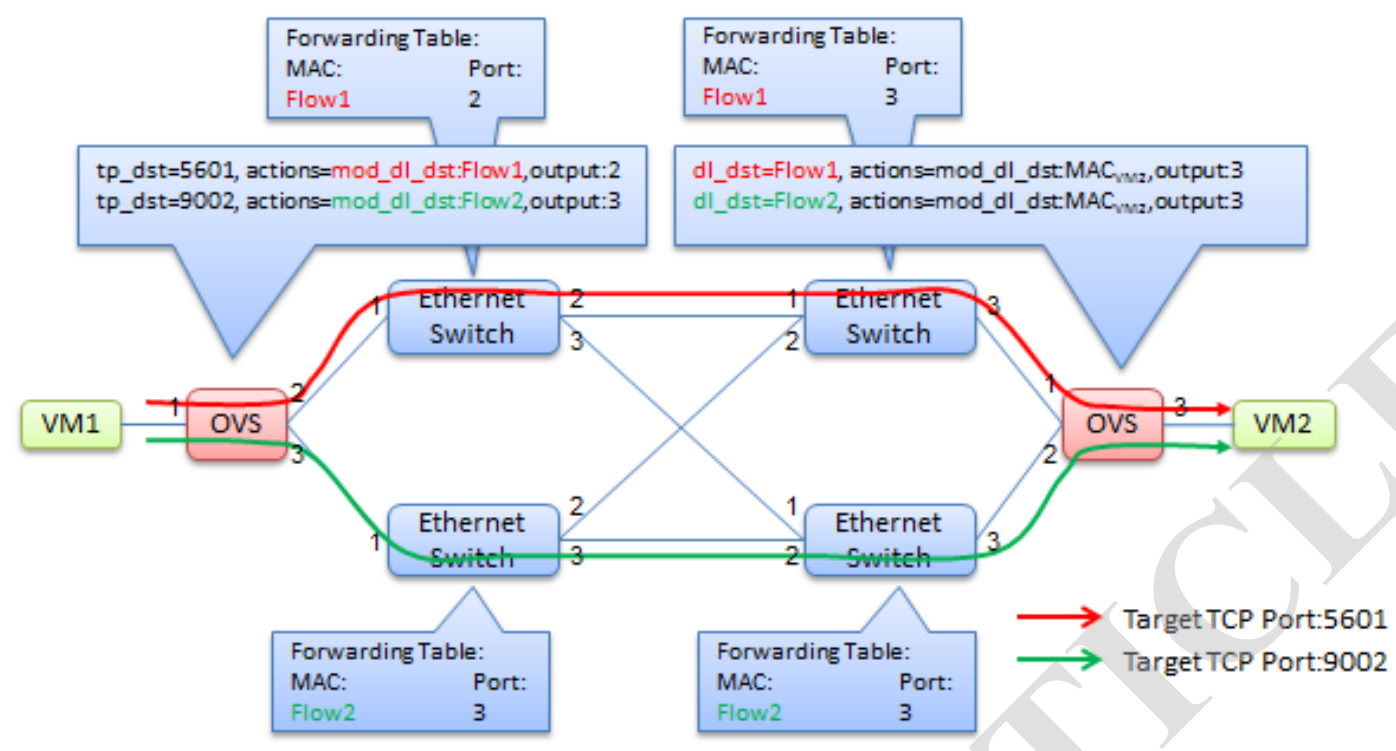

Fig. (6). The packets can be transmitted in terms of the flow ID.

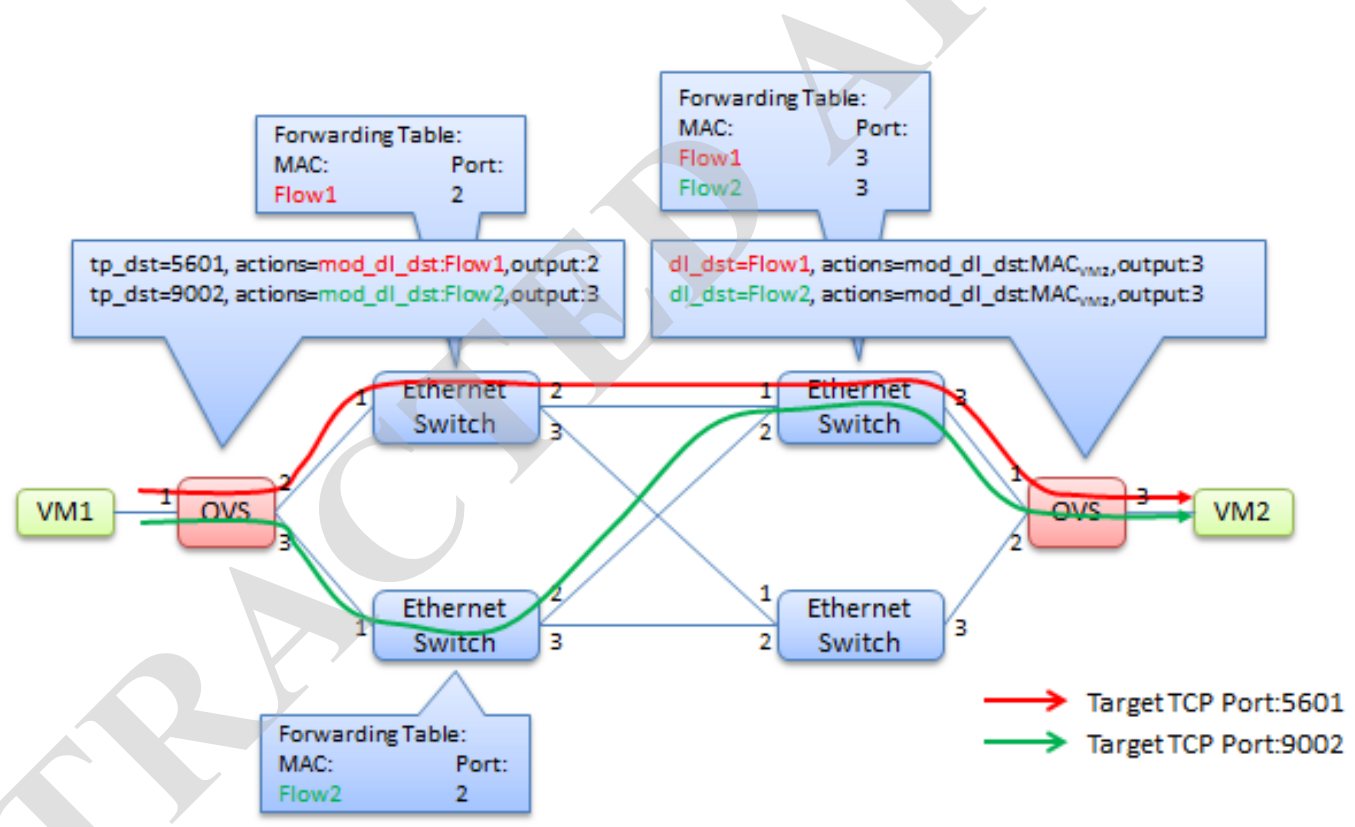

Fig. (7). Before the load balance of the dynamic flow by the ECOE.

and the packetshould be multicasted to the corresponding switch port. When the packet is multicasted to each corresponding Openflow, the second rule can deal with the packet. The second rule is that the VLAN ID should be removed and the packet should be transmitted to the VM port of the multicast when it receives the packet with the VLAN ID. In this way, the VMs belonging to the same multicast can communicate with each other through the multicast network address while other VMs can not receive the multicast packet. The function can adiot the Ethnernet to see the multicast MAC packets and the features multicasted by the same VLAN ID port. If the packet of all broadcast/multiast can be correlated with the belonging VLAN ID and the setting of the VLAN can be matched with each Ethernet, the function of freely controlling the the range of the broadcast/multicast packet/ However, the whole environment just can adopt the 4095 VLAN IDs which is a larger limitation. 


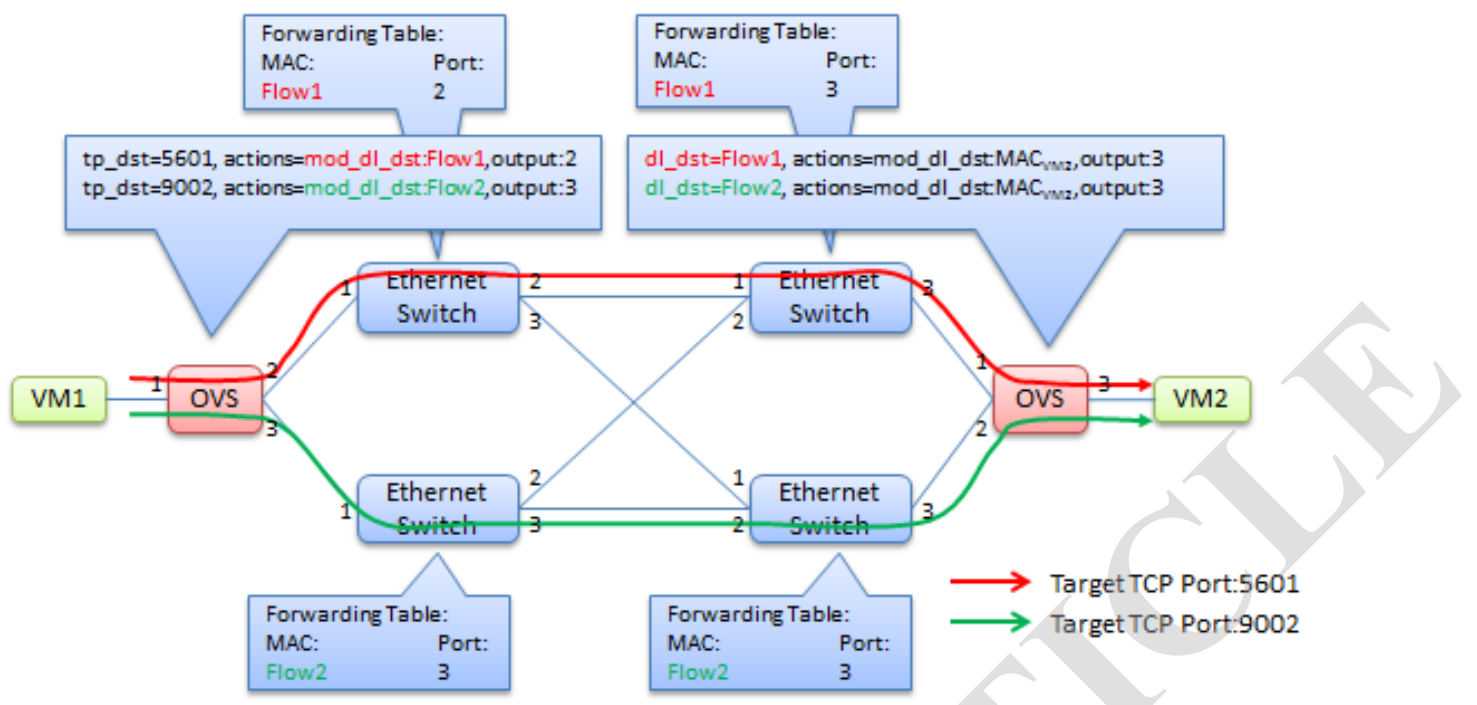

Fig. (8). After the load balance of the dynamic flow by the ECOE.

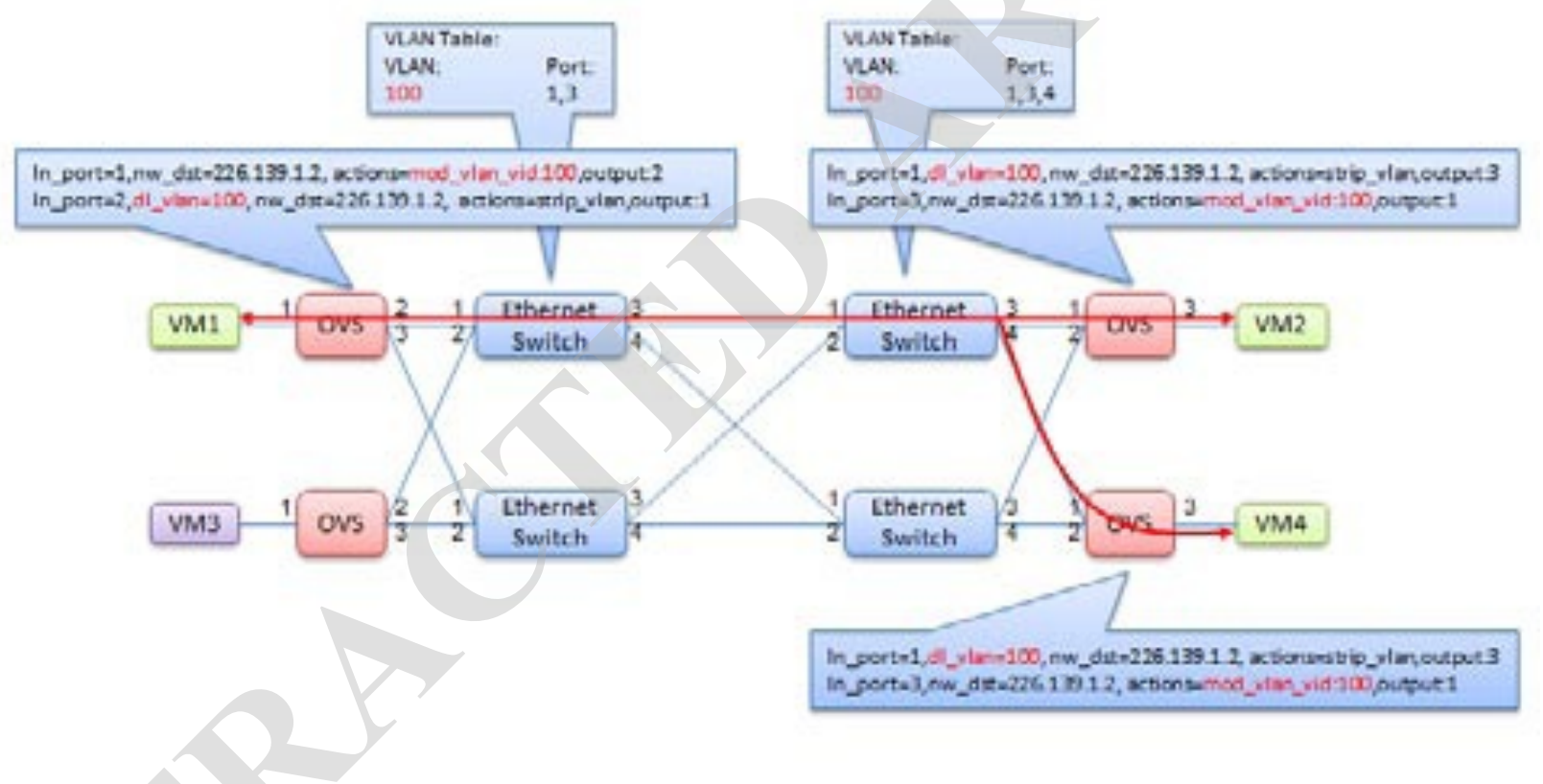

Fig. (9). The range control of the ECOE broadcast/multicast.

\section{THE VIRTUALIZATION AND THE SERVICE CHAIN OF THE NETWORK FUNCTION}

\subsection{The Virtualization of the Network Function}

Many devices of different Network Function are required in the Telecommunication companies of computer rooms and the service offered by the whole network function devices should be connected to provide users with a whole set of service, that is, yje concept of the network Service Chain. These devices are hoped to transmitted to the virtual host from the ardware and the transmitting rules written in the hardware of the service chain can be seperated in the issue of the Network Function Virtualization. In this way, the SDN is adopted to deal with all packet transmission and the busi- nessmen of offering the network service can be absorbed in developing the virtual network service, the SDN can be in charge of the packet transmission and the configuration of the service chain.

\subsection{The Service Chain of the Network Function}

The Fig. (10) is the Virtualization Network Function Forwarding Graph in which each VNF represents a Virtual Network Function and the telecommunication companies can help each users' online determine which network function should be passed to offer the service. The fold lines of the different colors in the Fig. (8) can pass the different virtual network function. These network function concatenated together can form a service chain. 


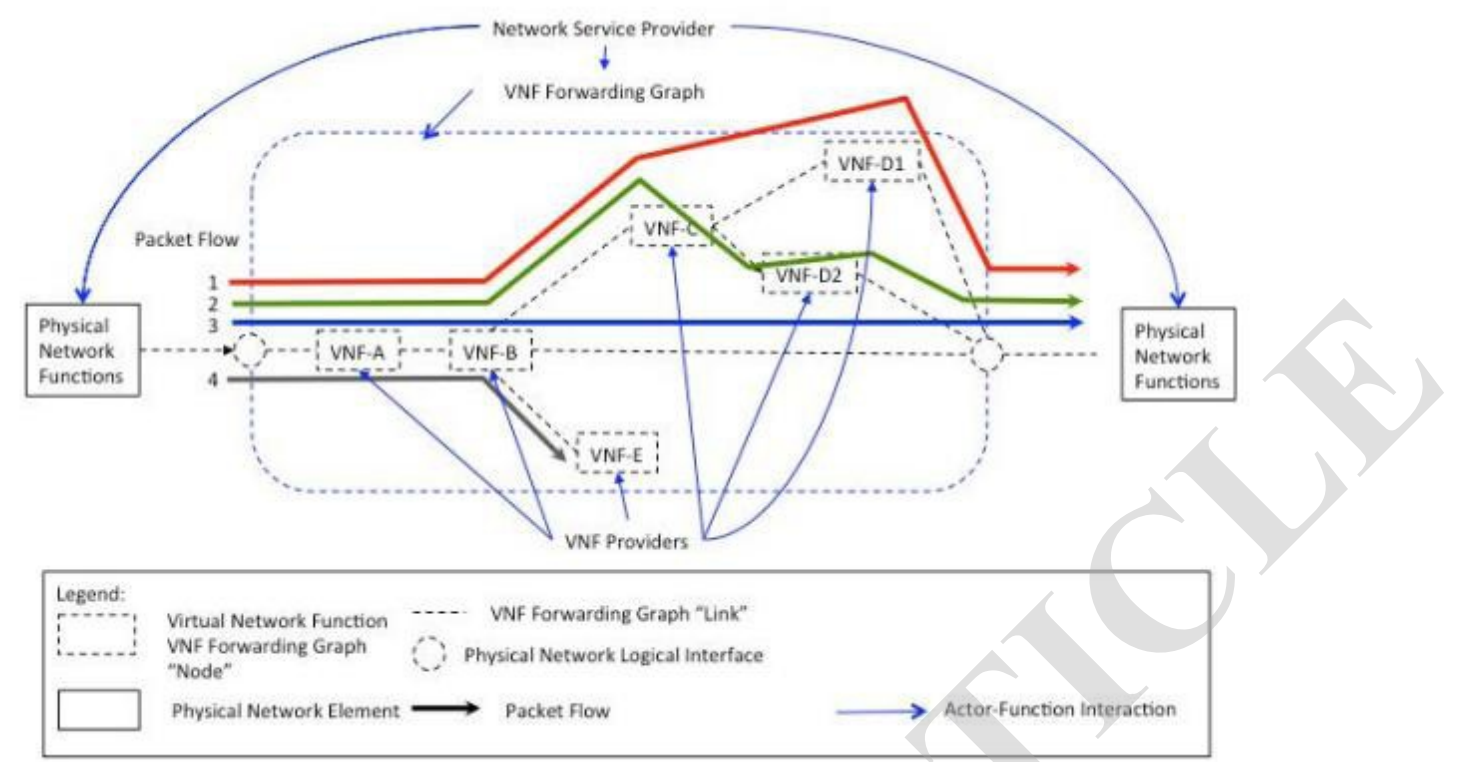

Fig. (10). The Virtualization Network Function Forwarding Graph.

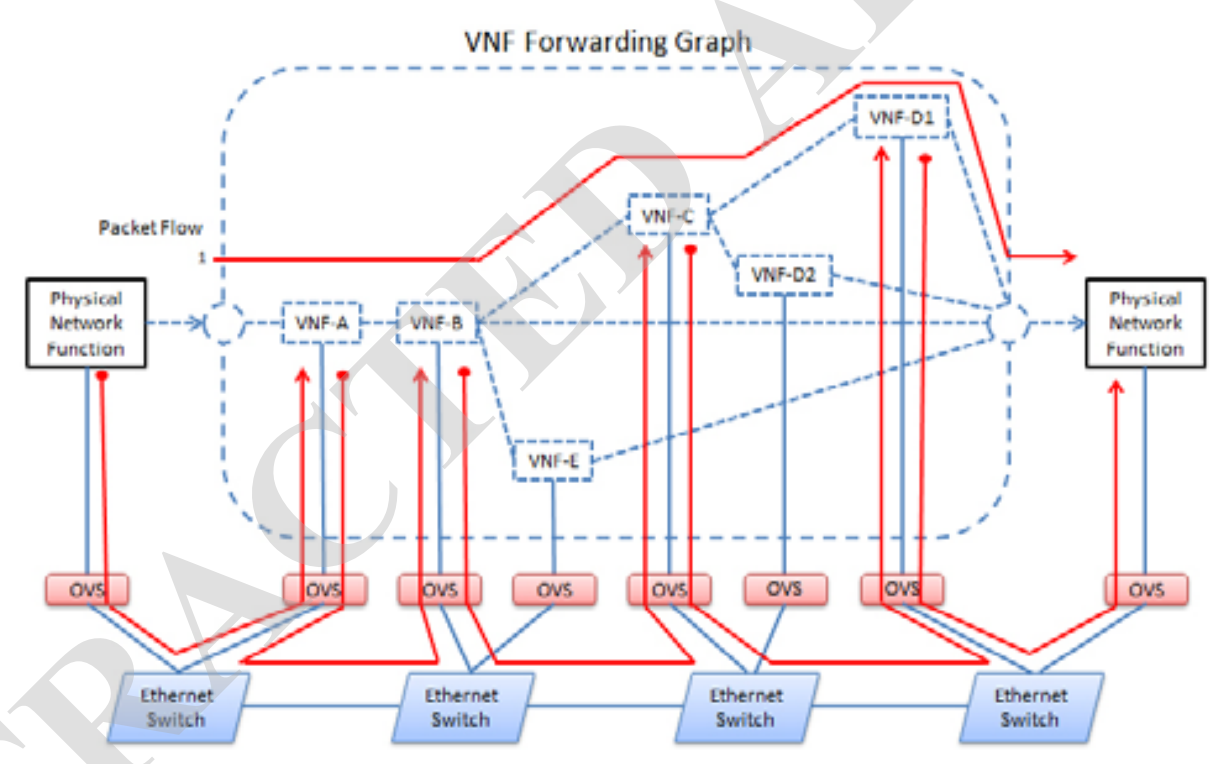

Fig. (11). The packet transmitting behavior in the service chain.

The Fig. (11) is the packet transmitting behavior in the service chain. If the Virtualization Network Function Forwarding Graph describes the online, it must pass the service chain formed by the four virtual network function VNF$\mathrm{A}, \mathrm{VNF}-\mathrm{B}, \mathrm{VNF}-\mathrm{C}$ and VNF-D1. The order is as follows, passing the VNF-A, the VNF-B, the VNF-C and the VNFD1. The actual transmission of the network is transmitting the packet to each virtual network function one station after another. Finally, the packets should be transmitted to the users. The part of it needs to depend on the SDN to set the network device for the request of the service chain in the upper layer definition. The specific kind of the packets can transmit the packet to the service node of eqach virtual network in terms of the order defined by the service chain. After the series connection in the service chain is finished, the packet can be transmitted. The service node in each virtual network can serve many service chains at the same time, maay be the beginning or the ending of a certain service chain.

\subsection{The Service Chain of the ECOE}

The Fig. (12) can achieve the method of the service chain in the ECOE environment. If the service chain should lock 


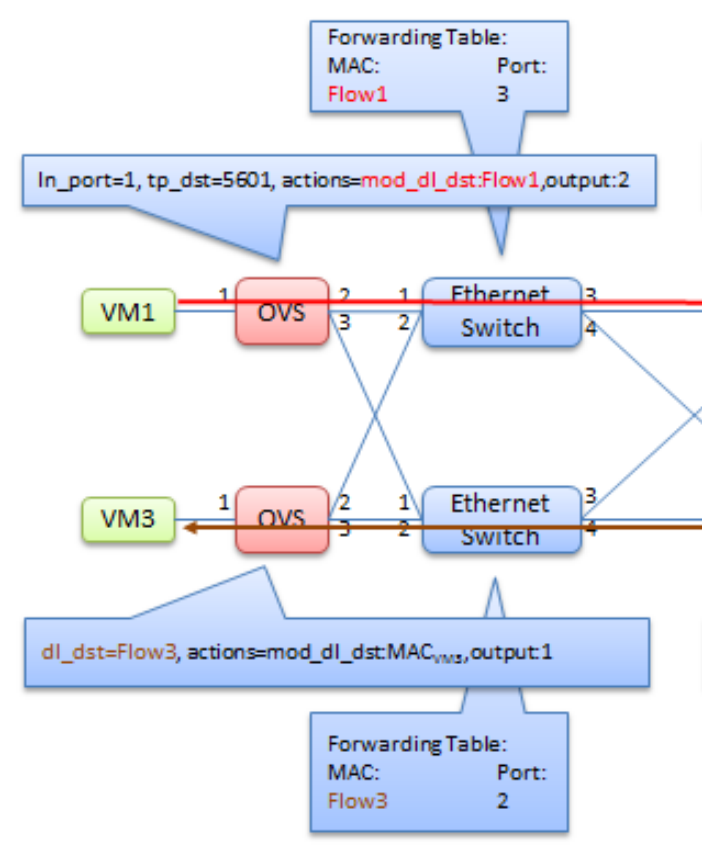

Fig. (12). The achieving method of the service chain in the ECOE.

the packet which should serve the target TCP port 5601, the passing sequence is the VM1, the VM2, the VM4 and the VM3. The service chain is connected into three parts. The first part is from the VM1 to the VM2. The second part is from the VM2 to the VM4. The third part is from the VM4 to the VM3. The method described in the third chapter should be adopted in the ECOE. The rules should be set in the source port of th Openflow in each station and the rule is that the flow ID can be given and transmitted to the next station if the target TCP port is 5601 and the packet is tranmistted from the specific VM. For example, the packet can be set as the flow 1 if it is transmitted from the VM1 and its target TCP port is 5601 . The packet can be set as the flow 2 if it is transmitted from the VM1 and its target TCP port is 5601. The packet can be set as the flow3 if it is transmitted from the VM4 and its target TCP port is 5601. The corresponding Static Forwarding Table in each flowID should be set in the Ethernet swithc and the packet can be transmitted to the corresponding VM one station after another so that the series connection of the whole service chain can be completed.

\section{CONCLUSION}

The network model of the ECOE proposed in the paper just needs to adopt to the Openflow around the network, and the present Ethernet in the core network can be used to offer the SDN function. The application of the network model can effectively help the Ethernet transfer the SDN. There is a trend that the intelligence of the SDN can be used around the network and the core part of the network just need to transmit the packet in the high speed. Therefore, the Openflow with the powerfun function can be put around the network. The core of the network adopts the high-speed Ethernet swithc to accord with the trend. The present Mellanox [9-11] also introduces the network card with the Openflow function so that each host can adopts to the Openflow in the future and the SDN function can be offered without changing all present Ethenet. Therefore, it is possible for the framework of the ECOE to adopt to the SDN network model.

\section{CONFLICT OF INTEREST}

The authors confirm that this article content has no conflicts of interest.

\section{ACKNOWLEDGEMENTS}

Declared none.

\section{REFERENCES}

[1] N. McKeown, T. Anderson, H. Balakrishnan, G. Parulkar, L. Peterson, J. Rexford, S. Shenker, and J. Turner, "OpenFlow: Enabling Innovation in Campus Networks," ACM SIGCOMM Computer Communication Review, USA, 2008.

[2] C. C. Tu, P. W. Wang, and T. C. Chiueh, "In-Band Control for an Ethernet-Based Software-Defined Network," ACM SYSTOR, USA, 2014.

[3] T. C. Chiueh, C. C. Tu, Y. C. Wang, P. W. Wang, K. W. Li, and Y. And M. Huang, "Peregrine: An All-Layer-2 Container Computer Network," In: Proc. IEEE Int. Conf. Cloud Computing, USA, 2012.

[4] C. Y. Lee, Y. W. Lee, C. C. Tu, P. W. Wang, Y. C. Wang, C. Y Lin, and T. C. Chiueh, "Autonomic Fail-over for a SoftwareDefined Container Computer Network," In: Proc. Int. Conf. Autonomic Computing, USA, 2013.

[5] N. Gude, T. Koponen, and J. Pettit, "NOX: Towards an Operating System for Networks," ACM SIGCOMM Computer Communication Review, USA, 2008.

[6] Floodlight OpenFlow controller [Online]. Available: http://www.projectfloodlight.org/floodlight/

[7] OpenDaylight, OpenDaylight Controller [Online]. Available: http://www.opendaylight.org/ 
[8] ITRI, ITRI Contribution to OpenDaylight: SNMP4SDN [Online]. Available:

https://wiki.opendaylight.org/view/Project_Proposals:SNMP4SDN

[9] OpenDaylight, Technical Overview [Online]. Available: http://www.opendaylight.org/project/technical-overview
[10] "Network Functions Virtualisation (NFV); Use Cases" [Online]. Available:

http://www.etsi.org/deliver/etsi_gs/NFV/001_099/001/01.01.01_60 /gs_NFV001v010101p.pdf

[11] Mellanox Technologies [Online]. Available:http://www.mellanox.com/_

Received: June 16, 2015

Revised: August 10, 2015

Accepted: September 19, 2015

(C) Chen et al.; Licensee Bentham Open.

This is an open access article licensed under the terms of the (https://creativecommons.org/licenses/by/4.0/legalcode), which permits unrestricted, non-commercial use, distribution and reproduction in any medium, provided the work is properly cited. 